Article

\title{
Application of Headspace Gas Chromatography-Ion Mobility Spectrometry for the Determination of Ignitable Liquids from Fire Debris
}

\author{
María José Aliaño-González, Marta Ferreiro-González * (D), Gerardo F. Barbero ${ }^{\circledR}$, Miguel Palma \\ and Carmelo G. Barroso \\ Department of Analytical Chemistry, Faculty of Sciences, ceiA3, IVAGRO, University of Cadiz, \\ 11510 Puerto Real, Cadiz, Spain; mariajose.alianogonzalez@alum.uca.es (M.J.A.-G.); \\ gerardo.fernandez@uca.es (G.F.B.); miguel.palma@uca.es (M.P.); carmelo.garcia@uca.es (C.G.B.) \\ * Correspondence: marta.ferreiro@uca.es; Tel.: +34-956-016-359
}

Received: 14 July 2018; Accepted: 7 August 2018; Published: 13 August 2018

\begin{abstract}
A fast and correct identification of ignitable liquid residues in fire debris investigation is of high importance in forensic research. Advanced fast analytical methods combined with chemometric tools are usually applied for these purposes. In the present study, the Headspace Gas Chromatography-Ion Mobility Spectrometry (HS-GC-IMS) combined with chemometrics is proposed as a promising technique for the identification of ignitable liquid residues in fire debris samples. Fire debris samples were created in the laboratory, according to the Destructive Distillation Method for Burning that is provided by the Bureau of Forensic Fire and Explosives. Four different substrates (pine wood, cork, paper, and cotton sheet) and four ignitable liquids of dissimilar composition (gasoline, diesel, ethanol, and paraffin) were used to create the fire debris. The Total Ion Current (TIC) Chromatogram combined with different chemometric tools (hierarchical cluster analysis and linear discriminant analysis) allowed for a full discrimination between samples that were burned with and without ignitable liquids. Additionally, a good identification ( $95 \%$ correct discrimination) for the specific ignitable liquid residues in the samples was achieved. Based on these results, the chromatographic data from HS-GC-IMS have been demonstrated to be very useful for the identification and discrimination of ignitable liquids residues. The main advantages of this approach vs. traditional methodology are that no sample manipulation or solvent is required; it is also faster, cheaper, and easy to use for routine analyses.
\end{abstract}

Keywords: headspace gas chromatography-ion mobility spectrometry; ignitable liquid residues; fire debris; multivariate analyses; total ion current chromatogram

\section{Introduction}

The identification of ignitable liquids (ILs) commonly used as accelerant by arsonists is a hard task for forensic analysts. Most of the ILs that are used as accelerants are petroleum-based products. So, the fast and continues development of petroleum industry and materials results in an increase of new liquids and new interference matrix that challenge even more the proper identification of the IL in fire debris analyses.

In addition, fire debris analysis is complicated due to many other reasons: the destructive nature of the fire, the high temperatures reached at the scene that usually evaporate almost all of the liquids, the use of foams and water by the firefighters [1,2], or the degradation phenomena, such as weathering or biodegradation that the samples can suffer after the fire [3-5].

For those reasons, it is necessary to keep investigating new reliable and rapid analytical methods that allow for the detection and identification of the IL [6,7]. A fast and proper identification of the IL 
allows for making decisions about the legal responsibilities of the incident $[8,9]$. Most of the researches about the identification and discrimination of both neat IL and ignitable liquid residues (ILRs) in fire debris that are described in the bibliography are based on gas chromatography-mass spectroscopy (GC-MS) [10,11]. Indeed, the American Society for Testing and Materials (ASTM) standard provides guidelines for the analysis of ILs from fire debris based on GC-MS [12-14]. The ASTM also provides a classification scheme in which the ILRs are classified into 8 major classes of IL: gasoline, petroleum distillates, isoparaffinic products, aromatic products, naphthenic-paraffinic products, normal alkane products, oxygenated solvents, and miscellaneous category [15]. Some databases, in particular, the Ignitable Liquids Database and Reference Collection (ILRC) created and maintained by the National Center for Forensic Science (NCFS) in collaboration with the Scientific Working Group for Fire and Explosions (SWGFEX), are available to assist fire debris analysts with the identification of ILRs form fire debris by following the ASTM E1618 [15] (https:/ /ilrc.ucf.edu/). The classification of each IL is based on visual pattern recognition of the total ion chromatograms (TICs), extracted ion profiling (EIPs), and target compound analysis [12,16,17].

So far, because ILRs are located fire debris solid samples, a first extraction method is usually required for a preconcentration of the samples before GC analysis. Different methods have been used with the purpose of separating the ILR from fire debris by passive headspace with different adsorbents, such as solid phase microextraction (SPME) fiber [14], Tenax [18], or activated carbon strips (ACS) [13]. Although these procedures work well, they still present some disadvantages, like the long adsorption times and the use of a solvent for desorbing the ACS, or the high price, the lowest robustness of the fibers as well as the lifetime of them in the case of SPME.

Regardless the preconcetration method, the analysis is done by GC-MS, and then, the interpretation of the results is based on the ASTM 1618 standard with the aim of classifying the ILR into one of the ASTM classes. The interpretation of the results is still a challenge for the analyst, since the approach is not only time consuming and complicated due to the interference matrix, moreover, it is highly related to the experience of the analyst running the analysis [19]. For this reason, in the last decade new alternatives or complementary approaches have been studied. The use of chemometric tools, in particular, pattern recognition techniques in order to obtain useful information of the total data matrix has been increased in the last years. The most commonly chemometric tools that are used are principal component analysis (PCA), cluster analysis (CA), discriminant analysis (DA), qualitative data analysis (QDA), or soft independent modelling by class analogy (SIMCA) [4,8,20,21].

The electronic nose (eNose) based on headspace concentration with mass spectrometry (HS-MS eNose) in combination with pattern recognition techniques has been successfully applied for direct analyses of ILR in fire debris samples in order to provide fast and almost fully automated results [6,7]. Headspace Gas Chromatography-Ion Mobility Spectrometry (HS-GC-IMS) uses the same sample preparation step than the eNose, i.e., headspace generation, and then, a gas sample is directly injected into the system. HS-GC-IMS is a novel technique with numerous advantages [22], as a high sensitivity (ppb range), fast analysis (a few minutes), option for portable devices, and no residues are generated. Due to these advantages, HS-GC-IMS has been applied in different fields as the oil industry [23,24], agrifood research [25-27], biomedical investigations [28,29], and in other forensic fields [30-32]. Nevertheless, it has not been previously applied for fire debris analyses.

The aim of the present work is to study the capacity of the HS-GC-IMS in combination with chemometrics tools (cluster analysis and discriminant analysis) for the determination and discrimination of different ILR in fire debris.

\section{Materials and Methods}

\subsection{Samples}

Four different substrates were chosen for this study (pinewood, cork, paper, and cotton sheet). All of the substrates were burned alone (without using any IL as accelerant) and then with each of the 
four ignitable liquids chosen for this study (gasoline, diesel, paraffin, and ethanol). Substrates and ILs were all purchased in local Spanish stores.

Fire debris samples were generated in the laboratory following Destructive Distillation Method for Burning provided by the Bureau of Forensic Fire and Explosives [17], inside of a laboratory hood with strict control of temperature $\left(25^{\circ} \mathrm{C}\right)$. For that, $5 \mathrm{~cm} \times 5 \mathrm{~cm}$ pieces of substrates were placed on the bottom of one-quart paint cans and $500 \mu \mathrm{L}$ of the corresponding ignitable liquid were added to the support. Cans were covered with a lid that contained nine $1 \mathrm{~mm}$ diameter holes.

Heat was applied to the bottom of the can with a flame from a propane torch held at a distance of $4 \mathrm{~cm}$ from the bottom. When smoke appeared, it was considered that the fire "starts" and samples were allowed to burn for two additional minutes. After this time, cans were allowed to cool down to room temperature and the perforated lids were replaced with an intact lid in order to avoid losing volatile compounds from the headspace.

Once the cans were cool, fire debris residues were extracted and placed into $10 \mathrm{~mL}$ vials (Agilent Crosslab) to their posterior analysis by HS-GC-IMS.

All of the experiments were carried out by duplicate and from each experiment; two pieces were obtained from different places of the can in order to guarantee no influence of the position of the support respect to the flame. A total of four burned samples were generated under the same conditions. So, a total of 80 fire debris samples were created.

\subsection{HS-GC-IMS Analysis Acquisition}

The 80 fire debris samples were analyzed by Headspace-Gas Chromatography-Ion Mobility Spectrometry FlavourSpec (G.A.S., Dortmund, Germany). No pretreatment was carried out for fire debris samples. Vials with fire debris were directly placed in the autosampler oven to be heated and agitated in order to generate headspace. Conditions for the creation of headspace were $75^{\circ} \mathrm{C}$ of incubation temperature for $20 \mathrm{~min}$ with agitation. A total of $200 \mu \mathrm{L}$ of headspace were injected with a splitless method of $500 \mu \mathrm{L} / \mathrm{s}$ while using a gas syringe at $80^{\circ} \mathrm{C}$, heated up to $5^{\circ} \mathrm{C}$ more than sampling temperature in order to avoid condensation phenomena. Between each sample injection, gas syringe was flushed with carried gas for five minutes to avoid cross-contamination. Conditions of analysis inside of HS-GC-IMS were as follows: column temperature of $40^{\circ} \mathrm{C}$ and IMS temperature of $45^{\circ} \mathrm{C}$. The drift gas flow was maintained at $150 \mathrm{~mL} / \mathrm{min}$ during the whole analyses. The initial carrier gas program started with a flow of $2 \mathrm{~mL} / \mathrm{min}$ for a minute, followed by a ramp carrier gas program of $5 \mathrm{~mL} / \mathrm{min}$ held for $4 \mathrm{~min}$ and then a flow of $150 \mathrm{~mL} / \mathrm{min}$, which was held for the final $10 \mathrm{~min}$ of the analysis. Gas chromatography column was multicapillary MCC OV-5 (G.A.S., Dortmund, Germany). Drift gas and carrier gas selected were nitrogen with a purity of $99.999 \%$, with a nitrogen generator (G.A.S., Dortmund, Germany). The ionization method used was by ${ }^{3} \mathrm{H}$ Tritium beta radiation.

\subsection{Data Analysis}

HS-GC-IMS implies a 2-dimensional separation of chemical compounds (retention time vs. drif time) as shown in Figure S1. In this case, two-dimensional chromatograms were obtained for each sample (intensity vs. retention time). They included information from 4286 retention time points from 0 to $899.85 \mathrm{~s}$, recording a data from each $0.21 \mathrm{~s}$. Total signal from the IMS system was used, meaning that no spectrometry information was used but the sum of signals as any drift time in the ion mobility spectrometer. Each chromatogram was normalized by assigning one unit to the maximum intensity. Information from the total ion current (TIC) chromatographic were arranged in data matrixes named $\mathrm{D}_{m \times n}$ where $m$ is the number of fire debris samples and $n$ is the number of retention times.

TIC chromatographic data were analyzed by chemometric tools. In particular, hierarchical cluster analysis (HCA) and linear discriminant analysis (LDA), while using the statistical computer package SPSS 22.0 (SPSS Inc., Armonk, NY, USA) were performed. 


\section{Results and Discussion}

Data from the total ion current (TIC) chromatograms were treated while using chemometric tools. No clear chromatographic peaks were obtained since very fast chromatographic analyses were run and the total signal from the IMS was used for this study. Therefore, chemometric tools were mandatory to extract the useful information from the TIC chromatograms. A hierarchical cluster analysis (HCA) using the whole data matrix $\left(\mathrm{D}_{80} \times 4286\right)$ was carried out. HCA is a non-supervised chemometric tool, and the purpose pursued was to search whether the differences detected in the TIC chromatograms were enough to detect and discriminate ILRs from fire debris samples. Ward's method and Squared Euclidian distance were used during the HCA. Results from these analyses were represented by a dendrogram displayed in Figure 1.

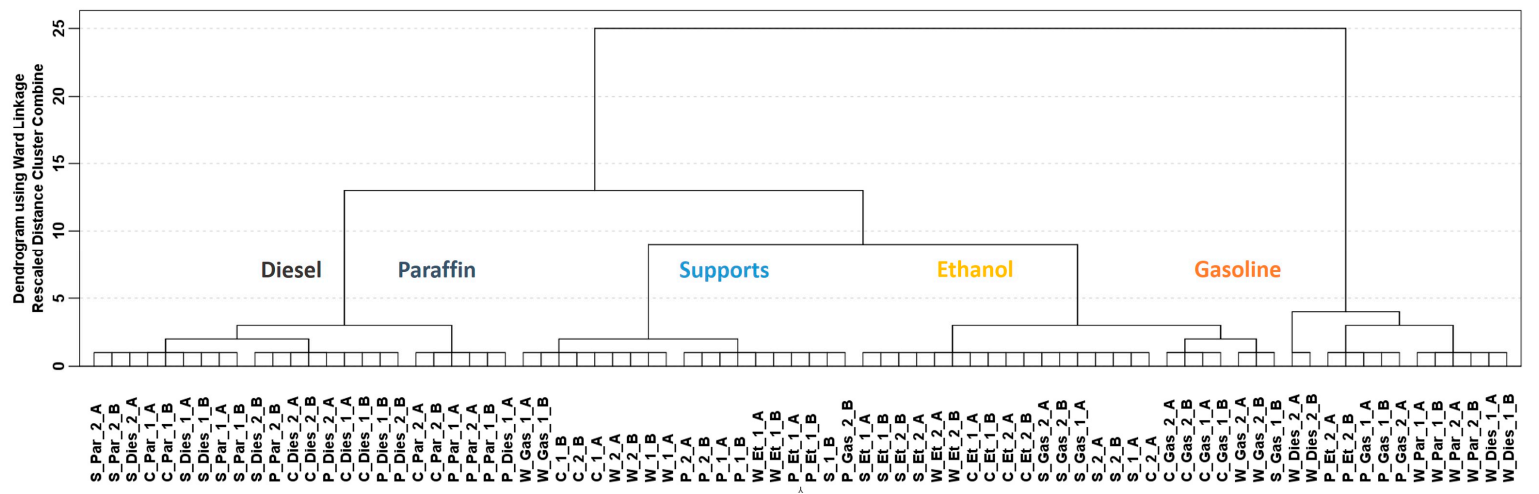

Figure 1. Dendrogram obtained from hierarchical cluster analysis (HCA) on the fire debris samples $\left(\mathrm{D}_{80} \times 4286\right)$ using TIC chromatograms. (Samples are denoted as the IL code followed by the substrate: Gasoline (Gas), Diesel (Dies), Paraffin (Par) and Ethanol (Et), cotton sheet (S), paper (P) for pinewood $(\mathrm{W})$ and for cork (C). A y B is used for the replicates.

Most of the supports burned without any IL (12 of 16 in total) are clustered together, as well as samples that were burned with ethanol (14 from 16). However, samples that were burned with diesel and paraffin are grouped in the same clusters. Diesel and paraffin are both heavy petroleum distillates so they have similar chemical composition, therefore they should provide similar related compounds to the ILR. Samples burned with gasoline are divided in two different clusters. These two groups of samples burned with gasoline as well as some of the misclassified samples seem to be related to the type of substrate used for burning and not to the IL used since all the cork and wood samples are clustered together regardless the IL. Although a full separation of the samples was not obtained, the results from this non-supervised technique suggest that the TIC chromatograms could be used to detect ILRs in fire debris or even to identify the ILRs.

Next, supervised pattern recognition tools to determine whether data from TIC chromatograms allow for the discrimination between samples was performed. For this purpose, a linear discriminant analysis (LDA) was carried out. A smoothing treatment for data was carried out and average of each ten columns was obtained by reducing the number of retention times to 428 . Two classes were established a priori, i.e., samples burned using ILs and samples burned without ILs.

During the LDA, $60 \%$ of the samples were randomly chosen as a calibration set and the remaining $40 \%$ of the samples were used to validate the model. Stepwise method for the LDA was applied. A full classification between the two groups of samples was achieved (100\%), first during the model development process, and later during the validation step. The score plot with all of the samples is represented in Figure 2. Samples that were used to calibrate the model were represented in blue in the case of the substrates burned alone and in yellow in the case of the ILRs. Samples that were used to validate the model were represented in a circle blue in the case of the supports burned alone and in a 
circle yellow in ILRs. A full discrimination between the two classes was obtained in the case of the calibration samples, as well as in the validation samples.

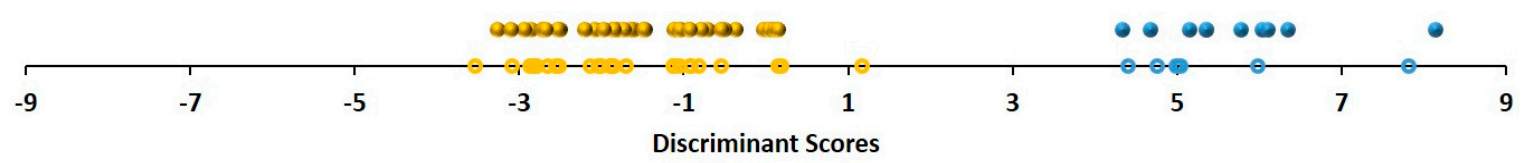

O Support burned without ILs (validation set)

- Support burned without ILs (calibration set)
- Support burned with ILs (validation set)

- Support burned with ILs (calibration set)

Figure 2. Discriminant scores obtained from linear discriminant analysis (LDA) for all of the fire debris samples $\left(D_{80} \times 428\right)$.

Fisher's linear discriminant functions were extracted from the LDA. A total of eight retention times showed a high contribution to those discriminant functions. Based on the selected retention times, average intensities for each retention time in the two groups were calculated and normalized to the maximum intensity inside of each group. In this way, two different fingerprints were generated (Figure 3), allowing for the discrimination between samples that were burned with IL and samples burned without IL. Therefore, the HS-GC-IMS based method can be used for the detection of ILR in fire debris.

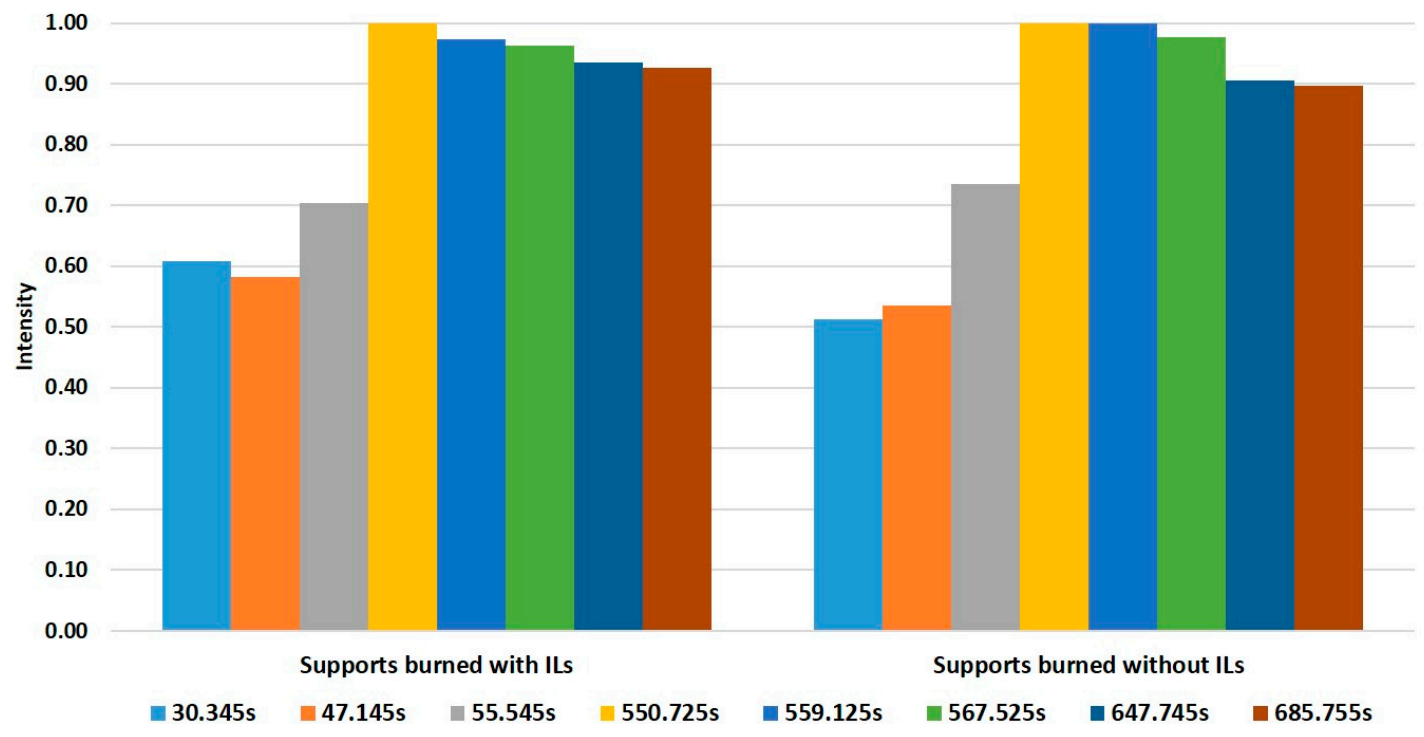

Figure 3. Fingerprints obtained from Fisher's linear discriminant function for samples burned with or without ILs.

Next, based on the results from the HCA, additional chemometric work was developed trying to discriminate between the different types of ILRs in the samples. A new LDA was carried out, but only using the samples that were burned with IL using again average retention times. Four groups were stablished (one for each kind of ILRs): gasoline (Gas_ILR), diesel (Die_ILR), ethanol (Et_ILR), and paraffin (Par_ILR). Cross-validation method (leave one out) and stepwise method was selected in the LDA. As shown in Table 1, a $98.4 \%$ of correct classification in the calibration set and a $98.4 \%$ for the cross-validated set was obtained. 
Table 1. Classification results from the LDA using the 64 samples burned with ignitable liquids (IL).

\begin{tabular}{|c|c|c|c|c|c|c|c|}
\hline \multicolumn{8}{|c|}{ Classification Results ${ }^{a, c}$} \\
\hline & & \multirow{2}{*}{ GR } & \multicolumn{4}{|c|}{ Predicted Group Membership } & \multirow{2}{*}{ Total } \\
\hline & & & Gas_ILR & Dies_ILR & Et_ILR & Par_ILR & \\
\hline \multirow{8}{*}{ Original } & \multirow{4}{*}{ Count } & Gas_ILR & 16 & 0 & 0 & 0 & 16 \\
\hline & & Dies_ILR & 0 & 15 & 0 & 1 & 16 \\
\hline & & Et_ILR & 0 & 0 & 16 & 0 & 16 \\
\hline & & Par_ILR & 0 & 0 & 0 & 16 & 16 \\
\hline & \multirow{4}{*}{$\%$} & Gas_ILR & 100.0 & 0 & 0 & 0 & 100.0 \\
\hline & & Dies_ILR & 0 & 93.8 & 0 & 6.3 & 100.0 \\
\hline & & Et_ILR & 0 & 0 & 100.0 & 0 & 100.0 \\
\hline & & Par_ILR & 0 & 0 & 0 & 100.0 & 100.0 \\
\hline \multirow{8}{*}{ Cross-validated ${ }^{\mathrm{b}}$} & \multirow{4}{*}{ Count } & Gas_ILR & 16 & 0 & 0 & 0 & 16 \\
\hline & & Dies_ILR & 0 & 15 & 0 & 1 & 16 \\
\hline & & Et_ILR & 0 & 0 & 16 & 0 & 16 \\
\hline & & Par_ILR & 0 & 0 & 0 & 16 & 16 \\
\hline & \multirow{4}{*}{$\%$} & Gas_ILR & 100.0 & 0 & 0 & 0 & 100.0 \\
\hline & & Dies_ILR & 0 & 93.8 & 0 & 6.3 & 100.0 \\
\hline & & Et_ILR & 0 & 0 & 100.0 & 0 & 100.0 \\
\hline & & Par_ILR & 0 & 0 & 0 & 100.0 & 100.0 \\
\hline
\end{tabular}

a $98.4 \%$ of original grouped cases correctly classified. ${ }^{\mathrm{b}}$ Cross validation is done only for those cases in the analysis. In cross validation. each case is classified by the functions derived from all cases other than that case. ${ }^{\mathrm{c}} 98.4 \%$ of cross-validated grouped cases correctly classified.

It can be observed that all misclassification occurs between groups of diesel and paraffin. During the model development, a sample burned with diesel was classified as sample burned with paraffin. Later, during the cross validation step, one sample burned with diesel was classified as being burned with paraffin again.

The score plot for all of the fire samples when using the three discriminant functions (FC1, FC2, and FC3) is displayed in Figure 4. It can be seen that, although they are not overlapped samples with diesel ILRs and paraffin, ILRs fell relatively close. As it was explained before, diesel and paraffin are chemically more similar in comparison to the other IL used in this study. FC3 is the function that shows higher influence for the discrimination between these two groups, while FC1 allows for the discrimination among samples that were burned with gasoline, with ethanol and the rest. Therefore, using the chromatographic data from the HS-GC-IMS, identifying the ILR in the fire debris is possible. However, a larger data set of fire debris samples would be needed to keep testing this novel technique, including several additional IL to follow the classification by the ASTM E1618.

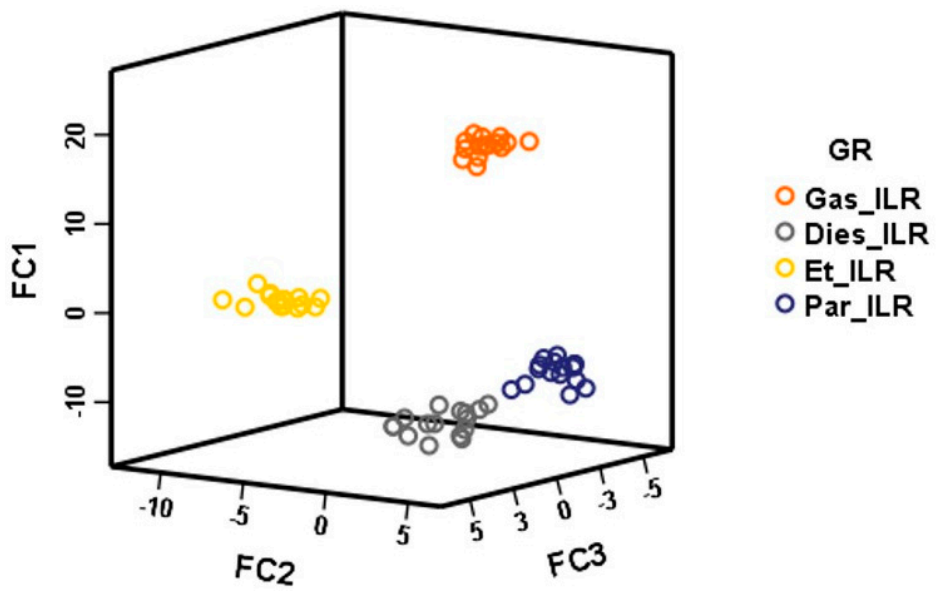

Figure 4. Discriminant scores obtained from LDA for burned samples $\left(D_{64} \times 428\right)$. 


\section{Conclusions}

It has been demonstrated that the results from the fire debris analysis by HS-GC-IMS allows for the detection of ILR in the samples. The four ILs that were used in this study can be detected in the fire debris samples using chemometric tools without sample preparation and in $15 \mathrm{~min}$.

Chemometric analysis of the HS-GC-IMS results also allows for the identification of the IL used among gasoline, diesel, paraffin, and ethanol with high reliability ( $98 \%$ good classification results).

Based on these results, HS-GC-IMS can be considered as a new promising tool in fire debris analyses. Besides, this technique has several advantages in comparison to traditional methods since HS-GC-IMS is a green technique (solvents are not required), fast, easy to use, and there are portable devices so the analyses could be applied at the fire scene.

Supplementary Materials: The following are available online at http:/ /www.mdpi.com/2297-8739/5/3/41/ s1, Figure S1: Example of 2-D plot (retention time vs. drif time) obtained for wood burned with gasoline by HS-GC-IMS.

Author Contributions: Data curation, M.J.A.-G. and M.P.; Formal analysis, M.F.-G. and G.F.B.; Investigation, M.J.A.-G. and M.F.-G.; Methodology, G.F.B. and M.P.; Resources, C.G.B.; Supervision, M.F.-G., G.F.B., M.P. and C.G.B.; Writing—original draft, M.J.A.-G.; Writing—review \& editing, M.F.-G. and M.P.

Funding: This research was funded by the University of Cadiz grant number PR2016-17.

Conflicts of Interest: The authors declare no conflict of interest.

\section{References}

1. Dennis, D.-M.K.; Williams, M.R.; Sigman, M.E. Investigative probabilistic inferences of smokeless powder manufacturers utilizing a bayesian network. Forensic Chem. 2017, 3, 41-51. [CrossRef]

2. Falatova, B.; Ferreiro-González, M.; Martín-Alberca, C.; Kačíková, D.; Galla, Š.; Palma, M.G.; Barroso, C. Effects of fire suppression agents and weathering in the analysis of fire debris by HS-MS eNose. Sensors 2018, 18, 1933. [CrossRef] [PubMed]

3. Martín-Alberca, C.; Carrascosa, H.; San Román, I.; Bartolomé, L.; García-Ruiz, C. Acid alteration of several ignitable liquids of potential use in arsons. Sci. Justice 2018, 58, 7-16. [CrossRef] [PubMed]

4. Martín-Alberca, C.; García-Ruiz, C.; Delémont, O. Study of chemical modifications in acidified ignitable liquids analysed by GC-MS. Sci. Justice 2015, 55, 446-455. [CrossRef] [PubMed]

5. Kindell, J.H.; Williams, M.R.; Sigman, M.E. Biodegradation of representative ignitable liquid components on soil. Forensic Chem. 2017, 6, 19-27. [CrossRef]

6. Ferreiro-Gonzalez, M.; Ayuso, J.; Alvarez, J.A.; Palma, M.; Barroso, C.G. Application of an HS-MS for the detection of ignitable liquids from fire debris. Talanta 2015, 142, 150-156. [CrossRef] [PubMed]

7. Ferreiro-Gonzalez, M.; Barbero, G.F.; Palma, M.; Ayuso, J.; Alvarez, J.A.; Barroso, C.G. Determination of ignitable liquids in fire debris: Direct analysis by electronic nose. Sensors 2016, 16, 695. [CrossRef] [PubMed]

8. Green, M.K.; Kuk, R.J.; Wagner, J.R. Collection and analysis of fire debris evidence to detect methamphetamine, pseudoephedrine, and ignitable liquids in fire scenes at suspected clandestine laboratories. Forensic Chem. 2017, 4, 82-88. [CrossRef]

9. Choi, S.; Yoh, J.J. Fire debris analysis for forensic fire investigation using laser induced breakdown spectroscopy. Spectrochim. Acta Part B Atomic Spectrosc. 2017, 134, 75-80. [CrossRef]

10. Martín-Alberca, C.; Ortega-Ojeda, F.E.; García-Ruiz, C. Analytical tools for the analysis of fire debris. A review: 2008-2015. Anal. Chim. Acta 2016, 928, 1-19. [CrossRef] [PubMed]

11. Sturaro, A.; Vianello, A.; Denti, P.; Rella, R. Fire debris analysis and scene reconstruction. Sci. Justice 2013, 53, 201-205. [CrossRef] [PubMed]

12. Stauffer, É.; Lentini, J.J. ASTM standards for fire debris analysis: A review. Forensic Sci. Int. 2003, $132,63-67$. [CrossRef] 
13. American Society for Testing and Materials (ASTM). Standard Practice for Separation of Ignitable Liquid Residues from Fire Debris Samples by Passive Headspace Concentration with Activated Charcoal; ASTM E1412 (2012); ASTM International: West Conshohocken, PA, USA, 2012.

14. American Society for Testing and Materials (ASTM). Standard Practice for Separation and Concentration of Ignitable Liquid Residues from Fire Debris Samples by Passive Headspace Concentration with Solid Phase Microextraction (SPME); ASTM E2154 (2008); ASTM International: West Conshohocken, PA, USA, 2001.

15. American Society for Testing and Materials (ASTM). Standard Test Method for Ignitable Liquid Residues in Extracts from Fire Debris Samples by Gas Chromatography-Mass Spectrometry; ASTM E1618 (2014); ASTM International: West Conshohocken, PA, USA, 2014.

16. Hupp, A.M.; Marshall, L.J.; Campbell, D.I.; Smith, R.W.; McGuffin, V.L. Chemometric analysis of diesel fuel for forensic and environmental applications. Anal. Chim. Acta 2008, 606, 159-171. [CrossRef] [PubMed]

17. Williams, M.R.; Sigman, M.E.; Lewis, J.; Pitan, K.M. Combined target factor analysis and bayesian soft-classification of interference-contaminated samples: Forensic fire debris analysis. Forensic Sci. Int. 2012, 222, 373-386. [CrossRef] [PubMed]

18. Borusiewicz, R.; Zadora, G.; Zieba-Palus, J. Application of headspace analysis with passive adsorption for forensic purposes in the automated thermal desorption-gas chromatography-mass spectrometry system. Chromatographia 2004, 60, 133-142. [CrossRef]

19. Lopatka, M.; Sigman, M.E.; Sjerps, M.J.; Williams, M.R.; Vivó-Truyols, G. Class-conditional feature modeling for ignitable liquid classification with substantial substrate contribution in fire debris analysis. Forensic Sci. Int. 2015, 252, 177-186. [CrossRef] [PubMed]

20. Sigman, M.E.; Williams, M.R. Assessing evidentiary value in fire debris analysis by chemometric and likelihood ratio approaches. Forensic Sci. Int. 2016, 264, 113-121. [CrossRef] [PubMed]

21. González-Rodríguez, J.; Sissons, N.; Robinson, S. Fire debris analysis by raman spectroscopy and chemometrics. J. Anal. Appl. Pyrolysis 2011, 91, 210-218. [CrossRef]

22. Li, F.; Xie, Z.; Schmidt, H.; Sielemann, S.; Baumbach, J.I. Ion mobility spectrometer for online monitoring of trace compounds. Spectrochim. Acta Part B Atomic Spectrosc. 2002, 57, 1563-1574. [CrossRef]

23. Garrido-Delgado, R.; del MarDobao-Prieto, M.; Arce, L.; Valcárcel, M. Determination of volatile compounds by GC-IMS to assign the quality of virgin olive oil. Food Chem. 2015, 187, 572-579. [CrossRef] [PubMed]

24. Garrido-Delgado, R.; Mercader-Trejo, F.; Sielemann, S.; de Bruyn, W.; Arce, L.; Valcárcel, M. Direct classification of olive oils by using two types of ion mobility spectrometers. Anal. Chim. Acta 2011, 696, 108-115. [CrossRef] [PubMed]

25. Vautz, W.; Baumbach, J.I.; Jung, J. Continuous monitoring of the fermentation of beer by ion mobility spectrometry. Int. J. Ion Mobil. Spectrom. 2004, 7, 3-5.

26. Camara, M.; Gharbi, N.; Cocco, E.; Guignard, C.; Behr, M.; Evers, D.; Orlewski, P. Fast screening for presence of muddy/earthy odorants in wine and in wine must using a hyphenated gas chromatography-differential ion mobility spectrometry (GC-DMS). Int. J. Ion Mobil. Spectrom. 2011, 14, 39-47. [CrossRef]

27. Márquez-Sillero, I.; Cárdenas, S.; Valcárcel, M. Direct determination of 2,4,6-tricholoroanisole in wines by single-drop ionic liquid microextraction coupled with multicapillary column separation and ion mobility spectrometry detection. J. Chromatogr. A 2011, 1218, 7574-7580. [CrossRef] [PubMed]

28. Mochalski, P.; Wiesenhofer, H.; Allers, M.; Zimmermann, S.; Güntner, A.T.; Pineau, N.J.; Lederer, W.; Agapiou, A.; Mayhew, C.A.; Ruzsanyi, V. Monitoring of selected skin- and breath-borne volatile organic compounds emitted from the human body using gas chromatography ion mobility spectrometry (GC-IMS). J. Chromatogr. B 2018, 1076, 29-34. [CrossRef] [PubMed]

29. Thompson, R.; Perry, J.D.; Stanforth, S.P.; Dean, J.R. Rapid detection of hydrogen sulfide produced by pathogenic bacteria in focused growth media using SHS-MCC-GC-IMS. Microchem. J. 2018, 140, 232-240. [CrossRef]

30. Armenta, S.; de la Guardia, M.; Alcalà, M.; Blanco, M.; Perez-Alfonso, C.; Galipienso, N. Ion mobility spectrometry evaluation of cocaine occupational exposure in forensic laboratories. Talanta 2014, 130, 251-258. [CrossRef] [PubMed] 
31. Keller, T.; Keller, A.; Tutsch-Bauer, E.; Monticelli, F. Application of ion mobility spectrometry in cases of forensic interest. Forensic Sci. Int. 2006, 161, 130-140. [CrossRef] [PubMed]

32. Moran, J.; McCall, H.; Yeager, B.; Bell, S. Characterization and validation of ion mobility spectrometry in methamphetamine clandestine laboratory remediation. Talanta 2012, 100, 196-206. [CrossRef] [PubMed]

(C) 2018 by the authors. Licensee MDPI, Basel, Switzerland. This article is an open access article distributed under the terms and conditions of the Creative Commons Attribution (CC BY) license (http://creativecommons.org/licenses/by/4.0/). 